\title{
Precision limits of tissue microstructure characterization by Magnetic Resonance Imaging
}

\author{
Analia Zwick, ${ }^{1,2}$ Dieter Suter, ${ }^{3}$ Gershon Kurizki, ${ }^{4}$ and Gonzalo A. Álvarez ${ }^{1,2,5, *}$ \\ ${ }^{1}$ Centro Atómico Bariloche, CONICET, CNEA, S. C. de Bariloche, Argentina. \\ ${ }^{2}$ Departamento de Física Médica, Instituto de Nanociencia y Nanotecnologia, CONICET, CNEA, S. C. de Bariloche, Argentina. \\ ${ }^{3}$ Fakultät Physik, Technische Universität Dortmund, D-44221, Dortmund, Germany \\ ${ }^{4}$ Chemical Physics Department, Weizmann Institute of Science, Rehovot, Israel \\ ${ }^{5}$ Instituto Balseiro, CNEA, Universidad Nacional de Cuyo, S. C. de Bariloche, Argentina.
}

\begin{abstract}
Characterization of microstructures in live tissues is one of the keys to diagnosing early stages of pathology and understanding disease mechanisms. However, the extraction of reliable information on biomarkers based on microstructure details is still a challenge, as the size of features that can be resolved with non-invasive Magnetic Resonance Imaging (MRI) is orders of magnitude larger than the relevant structures. Here we derive from quantum information theory the ultimate precision limits for obtaining such details by MRI probing of water-molecule diffusion. We show that already available MRI pulse sequences can be optimized to attain the ultimate precision limits by choosing control parameters that are uniquely determined by the expected size, the diffusion coefficient and the spin relaxation time $T_{2}$. By attaining the ultimate precision limit per measurement, the number of measurements and the total acquisition time may be drastically reduced compared to the present state of the art. These results will therefore allow MRI to advance towards unravelling a wealth of diagnostic information.
\end{abstract}

Information on compartment sizes and geometrical features of microstructures in live tissues is one of the potential keys to diagnosing tissue changes at early stages of pathologies and understanding organ malfunctioning due to diseases. For example, the biophysical mechanisms of cancer development and treatment are revealed by microstructure details [1-4]. Another case where small structural changes are important indicators are neuronal diseases that alter the distribution of axon diameters and myelin sheath, and thereby the speed of information propagation in the white matter of brain [5-10]. Such diseases include Alzheimer, autism, amyotrophic lateral sclerosis and schizophrenia. Therefore, a major goal of medical diagnosis is the development of precise and non-invasive techniques for characterizing the distribution of axon diameters in the brain and the sizes of microstructure compartments in tissues [11-13]. In order to find reliable biomarkers based on quantitative characterization of tissue microstructure, the diagnostic tools should provide precise measures of tissue structure size of the order of a few micrometers. For this purpose, it is not necessary to obtain micron-scale images of individual tissue compartments, but it is important to measure their average sizes at this resolution.

Magnetic resonance imaging (MRI) is an excellent tool for such studies, since it enables detailed, non-invasive characterization of tissues in vivo. Its resolution, in terms of voxel sizes, is typically limited to millimeters in clinical studies, or hundreds of microns in preclinical studies but reaching micrometer scales under specific conditions $[14,15]$. However, it also offers the potential to quantify structural details that are orders of magnitude smaller than the size of a voxel by monitoring the distance over which water molecules can travel by diffusion until their motion is restricted by walls that are not directly visible, such as cellular membranes. This approach is often called diffusion-weighted imaging (DWI) [16-19]. The most promising DWI technique employs Modulated Gradient Spin Echo (MGSE) sequences that enable detailed microstructure characterization [20-26]. Several works have addressed the estimation of compartment sizes by protocols based on various DWI sequences [23-30]. As pointed out by those works, the main open questions are: what resolution can be ultimately achieved by these experiments and how the experimental parameters should be adapted to approach this ultimate limit?

In this work, we provide answers to these important questions by adapting important results from quantuminformation theory: we analytically obtain this limit for the important case where the size of tissue microstructures are probed by diffusion processes via DWI experiments. We derive the necessary control conditions for MGSE sequences to allow the attainment of this limit. As examples, we consider sequences with typical modulated gradient waveforms for the estimation of microstructure compartment sizes, assuming generic geometries. We show that the ultimate precision limit of the estimation is achievable by MGSE sequences if the gradient strength is set to a value that depends on the microstructure size, the $T_{2}$ relaxation time and the diffusion coefficient of the molecules within the compartments. Based on this result, an optimization protocol is provided for MGSE sequences capable of attaining the highest possible precision under the given experimental constraints. With this protocol, the total acquisition time of quantitative microstructure imaging is shown to be drastically reduced, taking into account the limitations of the available hardware and the microscopic properties of the tissues being studied, such as diffusion constants and spin relaxation times.

Diffusion-weighted spin-echo NMR signal. Nuclear spins of molecules in biological tissues, in particular the spins $s=\frac{1}{2}$ of protons in water molecules, interact with 

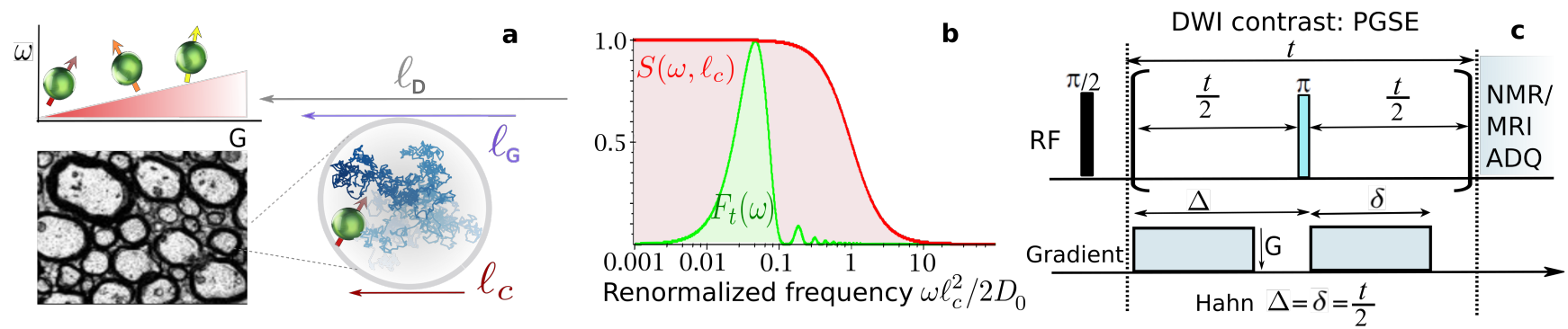

Figure 1. Diffusion-weighted spin-echo probing of length scales in tissue microstructure. (a) Schematic image of axon diameters in brain white matter. The myelin sheaths (dark circles) protect the cells of the central nervous system. In the presence of a magnetic field gradient, the Larmor frequency of the nuclear spins depends on their spatial positions. As the spins undergo Brownian motion, their displacement is limited by the distance between the walls of the compartment. Three relevant scales are compared: the restriction length $\ell_{c}$ is the main quantity that we have to characterize; it is related to the size of the compartment. The diffusion length $\ell_{D}$ is the average distance that the water molecules would travel in the case of free diffusion and $\ell_{G}$ is a dephasing length scale determined by experimental parameters that are sensitive to the diffusion process, such as the amplitude $G$ of the gradients. (b) Scheme of a normalized displacement power spectrum $S\left(\ell_{c}, \omega\right)$ (red color) and an optimal MGSE filter function $F_{t}(\omega)$ (green color) as a function of the renormalized frequency $\omega \ell_{c}^{2} / 2 D_{0}$. (c) Pulsed Gradient Spin Echo (PGSE) sequence for probing the diffusion restriction length: an initial $\frac{\pi}{2}$-excitation pulse is followed by the diffusion weighting-contrast building block that consists of a refocusing ( $\pi$ )-pulse at half the diffusion weighting time $t$ between two pulsed gradients of duration $\delta$ and separated by a delay $\Delta$. After the diffusion-weighting period, the remaining signal is measured, possibly using MRI encoding. The PGSE filter shown in panel $\mathbf{b}$, assumes $\delta=\Delta=\frac{t}{2}$.

external magnetic fields in MRI. A uniform magnetic field along the $z$ axis defines the Larmor precession frequency of the spins. In DWI, uniform magnetic-field gradients $G \hat{r}$ are applied along an arbitrary direction $\hat{r}$ (Fig. 1a). The spins are then subjected to fluctuating precession frequencies $\omega(t)$ induced by molecular diffusion displacements $[18,19]$. The instantaneous precession frequency of the spin $\omega(t)=\gamma G r(t)$ accounts for the random motion of molecular diffusion, where $r(t)$ is the instantaneous position of the diffusing spin along the field-gradient direction and $\gamma$ is the gyromagnetic factor of the nucleus. If the motion of the molecules is restricted, e.g. due to compartmentalization of the tissue, such as in the human brain, the fluctuations are limited in amplitude. Quantification of these fluctuations therefore allows one to obtain an indirect measure of the size distribution of these compartments as shown in Fig. 1a [11-13].

A typical MRI / DWI experiment starts with a $\pi / 2$ excitation pulse, which creates a coherent superposition of the two spin states. During the subsequent evolution within the diffusion time $t$, the spin-ensemble magnetization $M(t)=\left\langle e^{-i \phi(t)}\right\rangle M(0)$ records the diffusion process, where the brackets represent the ensemble average over the random phases. The total magnetization of the sample is encoded spatially by an MRI sequence at the end of this evolution. We consider here that $M(t)$ is the magnetization in a voxel of the image, that provides what is called the diffusion-weighted contrast. A Gaussian phase distribution is typically assumed for the random phase $\phi(t)$, leading, under probing by a MGSE sequence, to a decay of the magnetization Stepisnik [31]

$$
M(t)=e^{-\frac{1}{2}\left\langle\phi^{2}(t)\right\rangle} M(0),
$$

with the mean value $\langle\phi(t)\rangle=0$. Here, the signal attenuation factor $\frac{1}{2}\left\langle\phi^{2}(t)\right\rangle$ accounts for the contrast generated in an image, based on the effect of the MGSE sequence that probes different time scales of the diffusion process. The phase variance can be described in the frequency domain by the following expression [21, 32-34], which is the example for the case of phase-diffusion of the KofmanKurizki (KK) universal formula for decoherence (dephasing and relaxation) control and probing in quantum systems [34-40],

$$
\left\langle\phi^{2}(t)\right\rangle=\gamma^{2} \int_{-\infty}^{\infty} d \omega F_{t}(\omega) S(\omega) .
$$

The phase variance is a convolution of two spectral functions: (i) The filter function $F_{t}(\omega)$ is the finite-time Fourier transform (FT) of the magnetic field gradient (power spectrum) applied in the experimental sequence. It acts as a spectral noise-filter: If its value is 1 , it passes the experimental noise at that frequency without attenuation whereas if it is 0 , it blocks the noise completely. (ii) The spectral density $S(\omega)$ of the spin noise induced by the environment is given (in the case of diffusion) by the FT of the spin displacement autocorrelation function $\langle\Delta r(t) \Delta r(t+\tau)\rangle$, where $\Delta r(t)=r(t)-\langle r(t)\rangle$ is the instantaneous displacement deviation from the mean value [32, 33]. For molecular diffusion $\langle\Delta r(t) \Delta r(t+\tau)\rangle=D_{0} \tau_{c} e^{-|\tau| / \tau_{c}}$, where $D_{0}$ is the free diffusion coefficient $[23,29,41]$ and $\tau_{c}$ is the correlation time. For molecules that diffuse in a microstructure, the characteristic time $\tau_{c}$ is the one required on average for a molecule to probe the compartment boundaries. It is related to the restriction length by Einstein's expression $\ell_{c}^{2}=2 D_{0} \tau_{c}$ [19]. The spectral density 
$S(\omega)$ is given by $[20,33,37,40,41]$

$$
S(\omega)=\frac{D_{0} \tau_{c}^{2}}{\pi\left(1+\omega^{2} \tau_{c}^{2}\right)} .
$$

Figure $1 \mathrm{~b}$ shows $F_{t}(\omega)=\frac{1}{2 \pi}\left|\int_{0}^{t} d t^{\prime} G\left(t^{\prime}\right) e^{-i \omega t^{\prime}}\right|^{2}$ and $S(\omega)$ for a typical MGSE sequence displayed in Fig. 1c. In restricted diffusion, the specific relation between $\ell_{c}$ and the geometric size depends on the compartment shape (see Methods); e.g., for cylinders oriented perpendicular to the direction of the magnetic field gradient, a good approximation is $\ell_{c}=0.37 d$, where $d$ is the cylinder diameter [20, 21, 23, 32].

Ultimate error bounds for estimating microstructure sizes. The central question we pose is: What is the best MGSE control strategy to infer the restriction length of the diffusion process? To answer this question we resort to quantum information tools, in order to determine optimal gradient control strategies for obtaining the best estimation of the restriction length of the diffusion process, and thereby determine microstructure sizes in biological tissues.

The figure of merit for the estimation of $\ell_{c}$, the parameter that determines microstructure sizes, is the relative error $\frac{\delta \ell_{c}}{\ell_{c}}$. Assuming unbiased single-parameter estimation, the relative error of the magnetization signal in Eq. (1) is limited by the Cramer-Rao bound

$$
\frac{\delta \ell_{c}}{\ell_{c}} \geq \frac{\varepsilon\left(t, \ell_{c}\right)}{\sqrt{\mathcal{N}}}=\frac{1}{\ell_{c} \sqrt{\mathcal{N} \mathcal{F}_{\mathcal{Q}}\left(t, \ell_{c}\right)}},
$$

where $\varepsilon$ is the minimal attainable relative error per measurement, which is determined by the quantum Fisher information $(\mathrm{QFI}) \mathcal{F}_{\mathcal{Q}}\left(t, \ell_{c}\right)$ of $\ell_{c}$ obtainable from the measured spin and $\mathcal{N}$ is the number of measurements [42-44]. The QFI depends on the magnetization signal in Eq. (1) [40, 42, 45, 46] (see Methods) with a functional dependence on $\ell_{c}$ and the total diffusion weighting time $t$. This expression implicitly depends on other system parameters, particularly the diffusion coefficient $D_{0}$ that we assume to be known.

The relative error per measurement $\varepsilon$, Eq. (4), can be minimized by maximizing $\mathcal{F}_{\mathcal{Q}}$ with respect to the MGSE control parameters, specifically the gradient strength and modulation shape [40]. For a given MGSE sequence and a given gradient strength $G$, the optimal diffusion weighting time $t_{\text {opt }}$ is defined by

$$
\mathcal{F}_{\mathcal{Q}}\left(t_{\text {opt }}, \ell_{c}\right)=\max _{t}\left(\mathcal{F}_{\mathcal{Q}}\left(t, \ell_{c}\right)\right) .
$$

This define a minimal error $\varepsilon\left(t_{o p t}, \ell_{c}\right)$ for each MGSE control.

We can see that by suitably designing an optimal MGSE control, one can attain the ultimate relative-error bound for the restriction length of the diffusion process (see Methods), namely,

$$
\varepsilon\left(t, \ell_{c}\right) \geq \varepsilon_{0}
$$

Remarkably, this precision estimation bound for $\ell_{c}$ is general for all possible MGSE control sequences and independent of the particular geometry restricting the diffusion (see Methods). This ultimate precision is attained by optimally choosing the following length scales (Fig. 1a):

$$
\ell_{G}=\sqrt[3]{\frac{2 D_{0}}{\gamma G}} \quad \ell_{c}=\sqrt{2 D_{0} \tau_{c}} \quad \ell_{D}=\sqrt{2 D_{0} t},
$$

where $\ell_{G}$ the dephasing length, $\ell_{c}$ the restriction length, and $\ell_{D}$ is the the diffusion length.

Attaining the ultimate precision bound. To attain the ultimate error bound per measurement in Eq. (6), the MGSE should satisfy the following requirements: $(i)$ The spectral filter $F_{t}(\omega)$ should overlap with the displacement power spectrum $S\left(\ell_{c}, \omega\right)$ (Eq. (3)) within the spectral region of the highest power-law dependence on $\ell_{c}$ at a low frequency, where $S\left(\ell_{c}, \omega \approx 0\right) \propto \ell_{c}^{4}$, corresponding to $-\ln [M(t) / M(0)] \propto \ell_{c}^{4}$. (ii) The total diffusion weighting time $t$ should be the optimal time $t_{\text {opt }}$, Eq. (5), such that $\ln \left[M\left(t_{o p t}, \ell_{c}\right) / M(0)\right]=\ln M_{o}$, where the optimal magnetization contrast $M_{o}$ is determined by the expression $-\ln M_{o}=1+W\left(-2 e^{-2}\right) \approx 0.8$ (see Methods). A MGSE sequence producing a narrow low-frequency bandpass filter would be therefore the most sensitive to the restriction size $\ell_{c}$. The narrowest low-frequency bandpass filter for a given diffusion time among typical MGSE sequences is an optimized Pulse Gradient Spin Echo (PGSE) sequence, as it only contains one gradient-sign switch leading to the longest possible modulation period. The PGSE sequence [16] is displayed in Figure 1c. The time $\delta$ is the gradient pulse duration and $\Delta$ is the delay between the gradient pulses. The PGSE sequence includes one $\pi$ rf-pulse to refocus external magnetic field inhomogeneities. PGSE is analogous to a gradient echo or a Hahn spin echo [47] with a constant gradient if $\delta=\Delta=\frac{t}{2}$, where $t$ is the sequence duration and therefore the diffusion time (Fig. 1c) and produces the lowest frequency bandpass under this limit.

If $t \gg \tau_{c}$, the condition ( $i$ ) to attain the bound is satisfied resulting in (see Methods)

$$
-\ln \left(\frac{M_{\delta=\Delta=\frac{t}{2}, t \gg \tau_{c}}\left(t, \ell_{c}\right)}{M(0)}\right) \approx \gamma^{2} G^{2} D_{0} \tau_{c}^{2} t \propto \ell_{c}^{4} .
$$

From requirement (ii) and Eqs. (8), the optimal diffusion time is found to satisfy

$$
t_{\text {opt }}=\frac{-\ln M_{o}}{\gamma^{2} G^{2} D_{0} \tau_{c}^{2}} \approx \frac{0.8}{\gamma^{2} G^{2} D_{0} \tau_{c}^{2}} .
$$

Figure $2 \mathrm{a}$ shows typical signal decay for different $\gamma^{2} G^{2} D_{0} \tau_{c}^{3}$ values and the corresponding optimal diffusion times $t_{\text {opt }}$. Both requirements $(i)$ and (ii) are therefore fulfilled when $t=t_{\text {opt }} \gg \tau_{c}$, meaning that the diffusion length should be much larger than the correlation length, $\ell_{D} \gg \ell_{c}$ (Eq. 7). Together with Eq. (9), this requirement amounts to the condition $\gamma^{2} G^{2} D_{0} \tau_{c}^{3} \ll 1$ which 


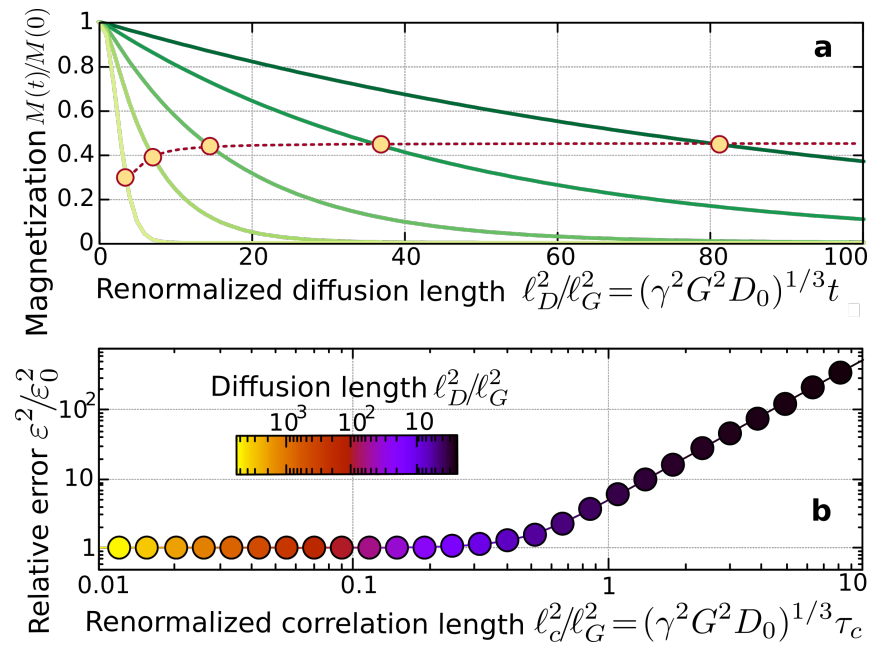

Figure 2. Attaining the ultimate precision bound of the restriction length $\ell_{c}$ from diffusion-weighted magnetization decay. (a) The decay of the normalized signal corresponding to the Hahn MGSE control $M(t) / M(0)$ as a function of the diffusion time in units of the square of the normalized diffusion length $\ell_{D}^{2} / \ell_{G}^{2}$, with $\ell_{D}^{2}=2 D_{0} t$. Dark to light green lines denote increasing renormalized correlation times in units of the renormalized restriction lengths $\ell_{c}^{2} / \ell_{G}^{2}=\left(\gamma^{2} G^{2} D_{0}\right)^{1 / 3} \tau_{c}=$ $0.1,0.15,0.25,0.4,1$. The circles denote the optimal diffusion times $\left(\gamma^{2} G^{2} D_{0}\right)^{1 / 3} t_{\text {opt }}$. (b) Relative minimum error $\frac{\varepsilon^{2}}{\varepsilon_{0}^{2}}$ in the estimation of $\ell_{c}$ per measurement, i.e. $\mathcal{N}=1$, as a function of the renormalized restriction length $\ell_{c}^{2} / \ell_{G}^{2}$ (colored circles). The ultimate error bound in the estimation of $\ell_{c}, \varepsilon_{0}$ in Eq. (6), is set at unity. The colored scale for the circles gives the normalized diffusion length $\ell_{D}^{2} / \ell_{G}^{2}$. The bound is attained when $\ell_{c}^{2} / \ell_{G}^{2} \ll 1$ and $\ell_{D}^{2} / \ell_{G}^{2} \gg 1$, and $\frac{\ell_{c}^{4}}{\ell_{G}^{4}} \frac{\ell_{D}^{2}}{\ell_{G}^{2}}=-\ln M_{0}$ as described in Eq. (9), implying $\left(\gamma^{2} G^{2} D_{0}\right)^{1 / 3} \tau_{c} \ll 1$. The quantity $\frac{\varepsilon^{2}}{\varepsilon_{0}^{2}}$ yields directly the number of measurements $\mathcal{N}$ needed to attain the ultimate precision bound per measurement.

means that the diffusion correlation length should be much smaller than the dephasing length, $\ell_{c} \ll \ell_{G}$ (see Fig. 1a). Indeed, the optimal diffusion-weighted length $\ell_{D} / \ell_{G}$ that corresponds to the highest precision per measurement for determining the restriction length, is seen from Fig. $2 b$ to require

$$
\frac{\ell_{c}^{6}}{\ell_{G}^{6}}=\gamma^{2} G^{2} D_{0} \tau_{c}^{3} \ll 1 .
$$

Therefore, under the idealized relaxation-free condition discussed here, increasing $\ell_{G}$, i.e. reducing the gradient strength, always improves the precision up to the point allowing to attain the bound (Eq. 6). Since the optimal time must fulfill Eq. (9), it must satisfy the power-law dependence $\frac{t_{\text {opt }}}{\tau_{c}}=\frac{\ell_{D}^{2}}{\ell_{G}^{2}} \frac{\ell_{G}^{2}}{\ell_{c}^{2}} \approx\left(\gamma^{2} G^{2} D_{0} \tau_{c}^{3}\right)^{-1}=\frac{\ell_{G}^{6}}{\ell_{c}^{6}} \gg 1$. By contrast, when $\frac{t_{o p t}}{\tau_{c}} \lesssim 1, t_{\text {opt }}$ saturates at a diffusion time value where the signal decays below $M / M(0) \approx 1 / e$ (Fig. 2a). The larger $\tau_{c}$, the shorter is $t_{\text {opt }}$ in Eq. (9), and the restricted diffusion regime $t \gg \tau_{c}$ can no longer be achieved.

Figure $2 \mathrm{~b}$ shows the minimal relative squared error $\varepsilon^{2}$ scaled to $\varepsilon_{0}^{2}$ per measurement, $\frac{\varepsilon^{2}}{\varepsilon_{0}^{2}}$. This scaled squared error determines the number of measurements $\mathcal{N}$ needed to attain an error equivalent to the ultimate precision per measurement (see Eq. (4)). For $\frac{\ell_{c}}{\ell_{G}}>1$, the restricted diffusion regime is no longer achieved and the relative error linearly increases with $\frac{\ell_{c}}{\ell_{G}}$.

Precision bounds with transverse relaxation. Under the idealized relaxation-free conditions discussed so far, by reducing the gradient we may always increase the optimal diffusion time so as to achieve the ultimate precision bound for $\ell_{c}$ estimation. However, this approach may fail, as the intrinsic nuclear-spin $T_{2}$-relaxation limits the accessible diffusion probing time. The $T_{2}$-relaxation contributes a global attenuation factor to the signal decay, which is independent of the MGSE sequence and the corresponding diffusion weighting. The echo signal of Eq. (1) is then $M_{T_{2}}\left(t, \ell_{c}\right)=e^{-\frac{t}{T_{2}}} M\left(t, \ell_{c}\right)$, where $M\left(t, \ell_{c}\right)$ accounts for the diffusion weighted spins' magnetization of Eq. (1). We can see that the relative error including the $T_{2}$-relaxation effects is bounded by

$$
\varepsilon \geq e^{\frac{t}{T_{2}}} \varepsilon_{0}
$$

and thus exponentially increases with $\frac{t}{T_{2}}$ (See Methods).

The conditions for attaining the ultimate error bound are now more restrictive, since the diffusion time $t$ cannot be larger than $T_{2}$. This condition implies specific values for the optimal diffusion time $t_{\text {opt }}$ and the efficiency parameter $\gamma^{2} G^{2} D_{0} \tau_{c}^{3}$ that can attain the best precision limit in the estimation of $\ell_{c}$. This is in contrast to the limit $T_{2} \rightarrow \infty$, where a semi-infinite range of $t_{\text {opt }}$ values exists for $\gamma^{2} G^{2} D_{0} \tau_{c}^{3} \ll 1$, that allow the ultimate error bound to be attained (see Fig. 2).

Yet, upon shortening $T_{2}$, while keeping $t_{\text {opt }} \ll T_{2}$ $\left(\ell_{D} \ll \ell_{T_{2}}=\sqrt{2 D_{0} T_{2}}\right.$ as Eq. (7)), we still find a finite region where $\varepsilon\left(t_{\text {opt }}, \ell_{c}\right) \approx \varepsilon_{0}$. This region is defined by the condition $\frac{\ell_{c}^{6}}{\ell_{G}^{6}} \ll 1$, Eq. (10), which has been imposed to satisfy the requirements for achieving the ultimate error bound. These two conditions imply that the ultimate error bound can be approached only if $\frac{\ell_{G}}{\ell_{T_{2}}} \ll 1$. Therefore the range of restriction lengths $\ell_{c}$ that can be determined efficiently with DWI must obey

$$
\left(\ell_{G} / \ell_{T_{2}}\right) \ell_{G}^{2} \ll \ell_{c}^{2} \ll \ell_{G}^{2},
$$

which are limited by the achievable gradient strengths and the $T_{2}$-relaxation time. For a given $\ell_{c}$, these conditions define the optimal gradients for estimating $\ell_{c}$

$$
\frac{1}{\gamma \ell_{c}^{2}} \sqrt{\frac{2 D_{0}}{T_{2}}} \ll G \ll \frac{2 D_{0}}{\gamma \ell_{c}^{3}} .
$$

The minimum relative error for different values of $G$ and $\ell_{c}$ is shown in Fig. 3, highlighting the optimal values of $G$ for estimating the allowed range of restriction lengths. 

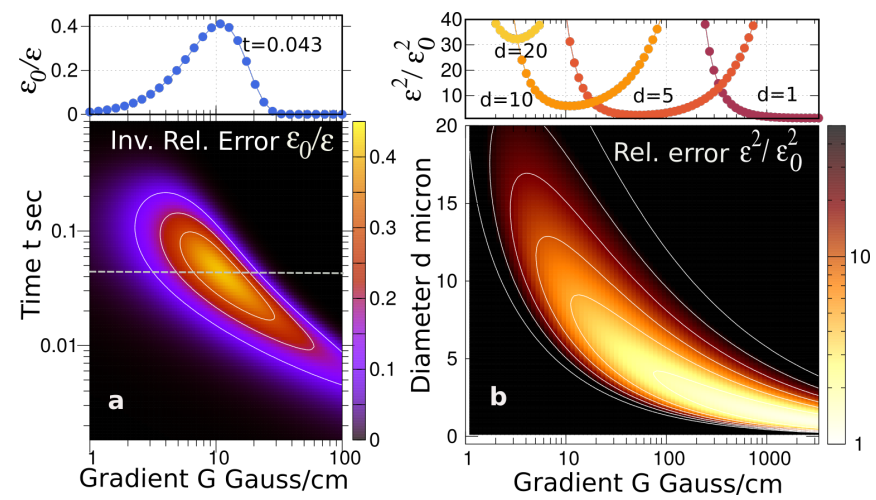

Figure 3. Attaining the precision bounds of the restriction length $\ell_{c}$ with transverse relaxation. (a) Effects of $T_{2}$ relaxation on the inverse of the estimation error $\frac{\varepsilon_{0}}{\varepsilon}$ in the estimation of $\ell_{c}$ per measurement, i.e. $\mathcal{N}=1$, highlighting the optimal time and gradient strength for a cylindrical compartment diameter of $d=10 \mu \mathrm{m}$. This geometrical shape is representative of axons or microfibers in general. The diffusion coefficient $D_{0}=1 \times 10^{-5} \frac{\mathrm{cm}^{2}}{\mathrm{~s}}$ and the relaxation time $T_{2}=0.1 \mathrm{~s}$ are typical of gray/white matter in the brain. The correlation length is $\ell_{c}=0.37 d$. The top inset shows the inverse of the estimation error $\frac{\varepsilon_{0}}{\varepsilon}$ as a function of the gradient $G$ for a diffusion time $t=43 \mathrm{~ms}$, shown by the white dashed line in the main panel. (b) The optimal squared relative error in the estimation of $d$ is normalized to the squared minimal error bound per measurement, $\varepsilon^{2} / \varepsilon_{0}^{2}$, is plotted as a function of the gradient $G$ and diameter $d$. The optimal gradient strengths are shown by brighter colors for a given diameter $d$. The relaxation time $T_{2}$ and the diffusion coefficient $D_{0}$ are considered as in a. The top inset shows the $\varepsilon^{2} / \varepsilon_{0}^{2}$ for $d=1,5,10,20 \mu \mathrm{m}$. The number of measurements required to attain $\varepsilon_{0}$ under the optimal conditions grows as the diameter $d$ increases or $T_{2}$ decreases. Equivalently, the range of optimal $G$ values is reduced as $\tau_{c}=\frac{1}{2} \ell_{c}^{2} / D_{0}$ approaches $T_{2}$ (Eq. 13).

Conclusions. Precise measurements of the size of microscopic tissue compartment, such as the diameter of neuronal axons is an ongoing endeavor that promises improved diagnostic value for many medical conditions. Diffusion of water molecules in these tissues provides a built-in tool that can be accessed by diffusion-weighted magnetic resonance techniques. The present analysis, which uses tools developed in quantum information science, reveals a universal, ultimate precision limit for estimating microstructure sizes by DWI. This limit is attainable by current MRI techniques available in many clinical settings, provided the relevant control parameters are properly chosen. We have shown here how the optimal parameters depend on the diffusion coefficient of the probe molecules (typically water), the relaxation time $T_{2}$, and the expected restriction length. The results of the present analysis are very encouraging as they show that optimal estimation of microstructure detail, e.g. axon diameters $(\sim 0.1-20 \mu \mathrm{m})$ is achievable by present technologies, given that modern clinical magnets can apply gradients of hundreds Gauss/cm [48], and preclinical micro-imaging magnet can apply thousands of
Gauss/cm. Even smaller microstructure sizes can, in principle, be determined with high precision, but this may demand higher field gradients that are currently unavailable in clinical magnets. The present results advance towards designing quantitative and precision imaging approaches opening new avenues for characterizing tissue microstructures in the shortest time possible, which is imperative to find useful biomarkers for medical diagnosis.

\section{Acknowledgment}

We thank L. Frydman and J. Jovicich for fruitful discussions. This work was supported by the EU FET Open PATHOS (G.K. and D.S.); DFG FOR 7024, ISF, QUANTERA PACE-IN (G.K.); CONICET, Instituto Balseiro and CNEA (A.Z., G.A.A.); ANPCyT-FONCyT PICT2017-3447, PICT-2017-3699, PICT-2018-04333, PIPCONICET (11220170100486CO), UNCUYO SIIP Tipo I 2019-C028 (A.Z., G.A.A.).

\section{METHODS}

Quantum Fisher Information on the microstructure size $\ell_{c}$. The quantum Fisher information (QFI) is given by [40, 42, 45, 46]

$$
\mathcal{F}_{\mathcal{Q}}\left(t, \ell_{c}\right)=\frac{\left[\frac{M\left(t, \ell_{c}\right)}{M(0)}\right]^{2}}{1-\left[\frac{M\left(t, \ell_{c}\right)}{M(0)}\right]^{2}}\left(\frac{\partial \ln \left[\frac{M\left(t, \ell_{c}\right)}{M(0)}\right]}{\partial \ell_{c}}\right)^{2},
$$

with explicit functional dependence on $\ell_{c}$ and the total diffusion weighting time $t$, but implicitly depends on the diffusion coefficient $D_{0}$ that we assume to be known, determined by monitoring the free diffusion time scale.

Ultimate error bound on $\ell_{c}$. The QFI is maximized at $t_{\text {opt }}$ which provides the best tradeoff between the amplitude contrast of the diffusion-weighted echo-signal $\left(M^{2}\left(M^{2}(0)-M^{2}\right)^{-1}\right)$ and its parametric-sensitivity to $\ell_{c},\left|\frac{\partial \ln (M / M(0))}{\partial \ell_{c}}\right|^{2}$. This parametric-sensitivity depends on $\frac{\partial S}{\partial \ell_{c}}$ since the correlation length $\ell_{c}$ only appears in the displacement power spectrum, Eq. (3). It is bounded by $\left|\frac{\partial S}{\partial \ell_{c}}\right| \leq \frac{4 S}{\ell_{c}}$. This bound is only reached when low frequencies are probed by the MGSE filter function. The spectrum in Eq. (3) then becomes an homogeneous function of $\ell_{c}$ of order 4 , i.e. $S\left(\ell_{c}, \omega \approx 0\right) \propto \ell_{c}^{4}$. This dependence leads to the bound

$$
\left|\frac{\partial \ln (M / M(0))}{\partial \ell_{c}}\right| \leq \frac{4(-\ln (M / M(0)))}{\ell_{c}},
$$

which is attained when the MGSE control is such that it generates a narrow low-frequency bandpass filter. This 
analysis determines a tight lower bound for the relative error in Eq. (4)

$$
\varepsilon\left(t, \ell_{c}\right) \geq \frac{\sqrt{[M(0)]^{2}-\left[M\left(t, \ell_{c}\right)\right]^{2}}}{4\left(-\ln \left[M\left(t, \ell_{c}\right) / M(0)\right]\right) M\left(t, \ell_{c}\right)},
$$

which is minimized when the condition

$$
-\ln \left[\frac{M\left(t, \ell_{c}\right)}{M(0)}\right]=-\ln M_{o}=1+\frac{W\left(-2 e^{-2}\right)}{2} \approx 0.8
$$

is fulfilled, with $W(z)$ being the Lambert function. This demonstrate the existence of an attainable ultimaterelative-error bound for the restriction length of the diffusion process

$$
\varepsilon\left(t, \ell_{c}\right) \geq \frac{\sqrt{1-M_{o}^{2}}}{4\left(-\ln M_{o}\right) M_{o}}=\varepsilon_{0} \approx 0.62,
$$

where $\varepsilon_{0}=\frac{\left(-\frac{1}{2} W\left(-2 e^{-2}\right)\left(1+\frac{1}{2} W\left(-2 e^{-2}\right)\right)^{-\frac{1}{2}}\right.}{4} \approx \frac{2.48}{4} \approx 0.62$.

The $T_{2}$ attenuation factor $e^{-\frac{t}{T_{2}}}$ of the signal decay, introduces a crucial constraint for attaining the optimal diffusion time that leads to the ultimate precision bound of Eq. (6) in the estimation of $\ell_{c}$. The relative error is now bounded by

$$
\begin{aligned}
\varepsilon \geq \frac{\sqrt{M^{2}(0)-e^{-\frac{2 t}{T_{2}}} M^{2}}}{4(-\ln [M / M(0)]) e^{-\frac{t}{T_{2}}} M} \\
\geq e^{\frac{t}{T_{2}}} \frac{\sqrt{M^{2}(0)-M^{2}}}{4(-\ln [M / M(0)]) M},
\end{aligned}
$$

which leads to Eq. (11).

Optimized PGSE: Hahn Spin-Echo Filter. The narrowest low-frequency bandpass filter from typical MGSE sequences is an optimized version of the PGSE sequence, as it only contains one gradient sign switch, producing the lowest frequency bandpass. The frequency filter of PGSE is

$$
F_{\delta, \Delta}^{P G S E}(\omega)=\left|\frac{4 i e^{-\frac{\mathrm{i} \omega(\delta+\Delta)}{2}} \sin \left(\frac{\omega \delta}{2}\right) \sin \left(\frac{\omega \Delta}{2}\right)}{\omega}\right|^{2} .
$$

For $\delta=\Delta=\frac{t}{2}$, which correspond to the well known gradient- or Hahn spin-echo refocusing sequences over the total diffusion time $t$ (Fig. 1), the magnetization signal is

$$
M_{\delta=\frac{t}{2}, \Delta=\frac{t}{2}}=e^{-\gamma^{2} G^{2} D \tau_{c}^{2} t\left[1-\frac{t}{\tau_{c}}\left(3+e^{-\frac{t}{\tau_{c}}}-4 e^{-\frac{t}{2 \tau_{c}}}\right)\right]} M(0) .
$$

Under this conditions, the sequence produces a lowfrequency narrow bandpass filter if $t \gg \tau_{c}$, resulting in Eq. (8).
Estimating restriction lengths in general geometries. Remarkably, the precision estimation bound for $\ell_{c}$ is general for all possible MGSE control sequences and independent of the particular geometry restricting the diffusion. The spectral density is the Fourier Transform of the diffusion correlation function derived from the solution to the Einstein-Fick diffusion equation for a corresponding restricting geometry $[20,31]$. The spectral density is therefore given by

$$
S(\omega)=\sum_{k} \frac{D_{0} b_{k} \tau_{k}^{2}}{\pi\left(1+\omega^{2} \tau_{k}^{2}\right)},
$$

where the coefficients $b_{k}$ and correlation times $\tau_{k}$ depend on the geometry of the compartments and can be found in Ref. [20] for cylinders, spheres and planar layers. When the MGSE filter overlaps with the displacement power spectrum $S(\omega)$ within the spectral region of low frequency $S\left(\ell_{c}, \omega \approx 0\right)=\sum_{k} \frac{D_{0} b_{k} \tau_{k}^{2}}{\pi} \propto \ell_{c}^{4}$. Here the restriction length is determined by the root mean squared correlation time $\ell_{c}^{2}=2 D_{0} \sqrt{\sum_{k} b_{k} \tau_{k}^{2}}$.

*gonzalo.alvarez@cab.cnea.gov.ar

[1] D. M. Patterson, A. R. Padhani, and D. J. Collins, Nat. Rev. Clin. Oncol. 5, 220 (2008).

[2] A. R. Padhani, G. Liu, D. M. Koh, T. L. Chenevert, H. C. Thoeny, T. Takahara, A. Dzik-Jurasz, B. D. Ross, M. Van Cauteren, D. Collins, D. A. Hammoud, G. J. S. Rustin, B. Taouli, and P. L. Choyke, Neoplasia 11, 102 (2009).

[3] N. S. White, C. R. McDonald, N. Farid, J. Kuperman, D. Karow, N. M. Schenker-Ahmed, H. Bartsch, R. RakowPenner, D. Holland, A. Shabaik, A. Bjørnerud, T. Hope, J. Hattangadi-Gluth, M. Liss, J. K. Parsons, C. C. Chen, S. Raman, D. Margolis, R. E. Reiter, L. Marks, S. Kesari, A. J. Mundt, C. J. Kaine, B. S. Carter, W. G. Bradley, and A. M. Dale, Cancer Res. 74, 4638 (2014).

[4] C. Enzinger, F. Barkhof, O. Ciccarelli, M. Filippi, L. Kappos, M. A. Rocca, S. Ropele, À. Rovira, T. Schneider, N. de Stefano, H. Vrenken, C. Wheeler-Kingshott, J. Wuerfel, F. Fazekas, and o. and, Nat. Rev. Neurol. 11, 676 (2015).

[5] J. B. Hursh, Am. J. Physiol. 127, 131 (1939).

[6] S. G. Waxman and M. V. L. Bennett, Nature New Biol. 238, 217 (1972).

[7] V. Drago, C. Babiloni, D. Bartrés-Faz, A. Caroli, B. Bosch, T. Hensch, M. Didic, H.-W. Klafki, M. Pievani, J. Jovicich, L. Venturi, P. Spitzer, F. Vecchio, P. Schoenknecht, J. Wiltfang, A. Redolfi, G. Forloni, O. Blin, E. Irving, C. Davis, H.-g. Hårdemark, and G. B. Frisoni, J. Alzheimers Dis. 26, 159 (2011).

[8] N. S. White, T. B. Leergaard, H. D’Arceuil, J. G. Bjaalie, and A. M. Dale, Hum. Brain Mapp. 34, 327 (2013).

[9] J. Xu, H. Li, K. D. Harkins, X. Jiang, J. Xie, H. Kang, M. D. Does, and J. C. Gore, Neuroimage 103, 10 (2014).

[10] F. Grussu, T. Schneider, C. Tur, R. L. Yates, M. Tachrount, A. Ianuş, M. C. Yiannakas, J. Newcombe, H. Zhang, D. C. 
Alexander, G. C. DeLuca, and C. A. M. Gandini WheelerKingshott, Ann. Clin. Transl. Neurol. 4, 663 (2017).

[11] Y. Assaf, T. Blumenfeld-Katzir, Y. Yovel, and P. J. Basser, Magn. Reson. Med. 59, 1347 (2008), https://onlinelibrary.wiley.com/doi/pdf/10.1002/mrm.21577.

[12] D. C. Alexander, P. L. Hubbard, M. G. Hall, E. A. Moore, M. Ptito, G. J. Parker, and T. B. Dyrby, Neuroimage 52, 1374 (2010).

[13] N. Shemesh, G. A. Álvarez, and L. Frydman, PLoS One 10, e0133201 (2015).

[14] J. Lambert, R. Hergenröder, D. Suter, and V. Deckert, Angew. Chem. Int. Ed. 48, 6343 (2009).

[15] E. Moore and R. Tycko, J. Magn. Reson. 260, 1 (2015).

[16] E. O. Stejskal and J. E. Tanner, J. Chem. Phys. 42, 288 (1965), https://doi.org/10.1063/1.1695690.

[17] D. Le Bihan, Nat. Rev. Neurosci. 4, 469 (2003).

[18] D. S. Grebenkov, Rev. Mod. Phys. 79, 1077 (2007).

[19] P. T. Callaghan, Translational Dynamics and Magnetic Resonance:Principles of Pulsed Gradient Spin Echo NMR (Oxford University Press, Oxford, 2011).

[20] J. Stepisnik, Physica B 183, 343 (1993).

[21] P. T. Callaghan and J. Stepisnik, J. Magn. Reson. 117, 118 (1995).

[22] P. T. Callaghan, J. Magn. Reson. 129, 74 (1997).

[23] N. Shemesh, G. A. Álvarez, and L. Frydman, J. Magn. Reson. 237, 49 (2013).

[24] I. Drobnjak, H. Zhang, A. Ianus, E. Kaden, and D. C. Alexander, Magn. Reson. Med. 75, 688 (2016), https://onlinelibrary.wiley.com/doi/pdf/10.1002/mrm.25631.

[25] M. Nilsson, S. Lasic, I. Drobnjak, D. Topgaard, and C.-F. Westin, NMR Biomed. 30, e3711 (2017), e3711 nbm.3711, https://onlinelibrary.wiley.com/doi/pdf/10.1002/nbm.3711.

[26] L. S. Kakkar, O. F. Bennett, B. Siow, S. Richardson, A. Ianus, T. Quick, D. Atkinson, J. B. Phillips, and I. Drobnjak, Neuroimage 182, 314 (2018), microstructural Imaging.

[27] H. H. Ong and F. W. Wehrli, Neuroimage 51, 1360 (2010).

[28] M. E. Komlosh, E. Ozarslan, M. J. Lizak, F. Horkay, V. Schram, N. Shemesh, Y. Cohen, and P. J. Basser, J. Magn. Reson. 208, 128 (2011).

[29] G. A. Álvarez, N. Shemesh, and L. Frydman, Phys. Rev. Lett. 111, 080404 (2013).

[30] J. Xu, X. Jiang, H. Li, L. R. Arlinghaus, E. T. McKin- ley, S. P. Devan, B. M. Hardy, J. Xie, H. Kang, A. B. Chakravarthy, and J. C. Gore, Magn. Reson. Med. , mrm.28056 (2019).

[31] J. Stepisnik, Physica B 270, 110 (1999).

[32] J. Stepisnik, S. Lasic, A. Mohorix, I. Sersa, and A. Sepe, J. Magn. Reson. 182, 195 (2006).

[33] S. Lasic, J. Stepisnik, and A. Mohoric, J. Magn. Reson. 182, 208 (2006).

[34] G. A. Álvarez and D. Suter, Phys. Rev. Lett. 107, 230501 (2011).

[35] A. Kofman and G. Kurizki, Nature 405, 546 (2000).

[36] A. G. Kofman and G. Kurizki, Phys. Rev. Lett. 87, 270405 (2001).

[37] A. G. Kofman and G. Kurizki, Phys. Rev. Lett. 93, 130406 (2004).

[38] G. Gordon, N. Erez, and G. Kurizki, J. Phys. B: At. Mol. Opt. Phys. 40, S75 (2007).

[39] G. Kurizki and A. Zwick, in "From coherent to incoherent dynamical control of open quantum systems", To appear in Adv. Chem. Phys. 159, edited by P. Brumer, S. A. Rice, and A. R. Dinner (John Wiley, 2015).

[40] A. Zwick, G. A. Álvarez, and G. Kurizki, Phys. Rev. Applied 5, 014007 (2016).

[41] J. R. Klauder and P. W. Anderson, Phys. Rev. 125, 912 (1962).

[42] M. G. A. Paris, International Journal of Quantum Information 07, 125 (2009).

[43] S. Braunstein and C. Caves, Phys. Rev. Lett. 72, 3439 (1994).

[44] H. Cramér, Mathematical methods of statistics (Princeton university press, 1946).

[45] C. Benedetti and M. G. Paris, Phys. Lett. A 378, 2495 (2014).

[46] G. Kurizki, E. Shahmoon, and A. Zwick, Phys. Scr. 90, 128002 (2015).

[47] E. Hahn, Phys. Rev. 80, 580 (1950).

[48] K. Setsompop, R. Kimmlingen, E. Eberlein, T. Witzel, J. Cohen-Adad, J. A. McNab, B. Keil, M. D. Tisdall, P. Hoecht, P. Dietz, S. F. Cauley, V. Tountcheva, V. Matschl, V. H. Lenz, K. Heberlein, A. Potthast, H. Thein, J. Van Horn, A. Toga, F. Schmitt, D. Lehne, B. R. Rosen, V. Wedeen, and L. L. Wald, Neuroimage Mapping the Connectome, 80, 220 (2013). 